\title{
THE PROCESS OF POROUS TANTALUM IMPLANTS OSSEOUS INTEGRATION A REVIEW
}

\author{
Christos G. Paganias, George A. Tsakotos, \\ Stephanos D. Koutsostathis and George A. Macheras \\ Department of Orthopaedics, KAT Hospital, 2, Nikis Str., Kifissia, Athens, Greece
}

Received 2012-05-01; Revised 2013-05-01; Accepted 2013-12-31

\begin{abstract}
Porous tantalum is a biomaterial that was recently introduced in orthopaedics in order to overcome problems of the past related to implant loosening. Its use has led to very good results, especially in difficult cases where severe bone defect is present, as it is found to have osteoconductive and possibly, osteoinductive properties. So, it is of great interest to shed light to the mechanisms through which this material leads to new bone formation after being implanted. For this reason, a review of the current literature was undertook. Porous tantalum is, biologically, relatively inert, meaning that its bonding capacity to the bone is restricted. In order to overcome this obstacle, it undergoes thermal processing in alkaline environment. This process leads to extensive hydroxyapatite formation on its surface and thus, to better integration of porous tantalum implants. Apart from this, new bone tissue formation occurs inside the pores of porous tantalum after its implantation and this new bone retains the characteristics of normal bone, i.e., bone remodeling and Haversian systems formation. This finding is enhanced by the observation that porous tantalum is an appropriate substrate for osteoblast adherence, proliferation and differentiation. Furthermore, the finding that osteoblasts derived from old women ( $>60$ years old) and cultivated on porous tantalum may grow faster than osteoblasts taken from younger women ( $<45$ years old) and cultivated on other substrates, can partially explain porous tantalum good performance in cases of patients with severe bone defects. In conclusion, porous tantalum chemical and mechanical properties are those that, probably, define the already noticed good performance of this material. However, further research is needed to totally clarify the mechanisms through which they contribute to that.
\end{abstract}

Keywords: Porous Tantalum, Osseous Integration, Bone Ingrowth, Osteoconductive, Osteoblast, Scaffold

\section{INTRODUCTION}

Porous tantalum is a relatively new biomaterial in orthopaedics, used in domains such as hip and knee arthroplasty, hip osteonecrosis surgery and spine surgery. It consists of a carbon scaffold on which pure tantalum is deposited. Porous tantalum possesses some unique mechanical properties, mainly due to its high porosity. It has a low modulus of elasticity, close to that of subchondral and cancellous bone, leading to better load transfer and minimized stress shielding phenomenon. Its coefficient of friction is among the highest when talking about biomaterials, allowing for sufficient primary stabilization of implants, even without screw fixation. These parameters are thought to contribute to the good and sometimes excellent, clinical results achieved with the use of porous tantalum. However, it is more interesting and probably, of even greater importance, to assess the processes taking place at the bone-porous tantalum interface, in terms of hydroxyapatite and bone tissue formation, not only on the surface, but also inside the biomaterial. Furthermore, it is equally important to examine the role of porous tantalum as a substrate for osteoblast proliferation and differentiation, as the procedure of porous tantalum integration takes place through new bone tissue formation and osteoblasts are the key cells for this process.

Corresponding Author: Christos G. Paganias, Department of Orthopaedics, KAT Hospital, 2, Nikis Str., Kifissia, Athens, Greece 


\subsection{Porous Tantalum Thermal Processing in Alkaline Environment}

Porous tantalum is biologically relatively inert (Levine et al., 2006), which means that it's bonding capacity to the bone is restricted. This kind of biologic activity is necessary when talking about a metal, in order to achieve an enhanced attachment to the surrounding soft tissues and also, to the underlying bone-especially when bone defect is present. For this reason, the development of biologically active tantalum is of great importance. Previous studies testing the formation of biologically active ceramics concluded that the most crucial point in order to achieve bone integration of the implants- is the formation of a biologically active hydroxyapatite layer on their surface after being implanted (Kotani et al., 1991). This can be achieved by hydroxyapatite plasma-spraying (Kitsugi et al., 1996). When this procedure takes place, the temperature of the hydroxyapatite powder may reach the value of $10000^{\circ} \mathrm{C}$, which leads to its partial melting and degradation. This results to the formation of a coating of different structure, if compared to hydroxyapatite. As a consequence, this coating may detach from the underlying bone (Geesink et al., 1995). In order to overcome these difficulties, a different procedure is needed for the formation of hydroxyapatite coating. This is achieved through the use of porous tantalum implants that have undergone thermal processing in an alkaline environment.

In accordance to this, an effort was made to form hydroxyapatite coating on porous tantalum surface. Through trials, it was observed that hydroxyapatite- like coating can be formed on amorphous tantalite hydrogel surface when it is prepared in a $0.2-0.5 \mathrm{M} \mathrm{NaOH}$ solution (Miyazaki et al., 2000; 2002). This layer gets stabilized after thermal treatment at $300^{\circ} \mathrm{C}$ and becomes a stable layer of amorphous tantalum salt (Kato et al., 2000; Kokubo, 2004). When impregnated to SBF (Simulated Body Fluid) - consisting of an acellular fluid with ion concentration similar to that of human serum- the substrate that has undergone treatment in an alkaline environment shows hydroxyapatite formation within one week (Kokubo et al., 1990; Kim et al., 1996). By the time sites of hydroxyapatite formation appear, they begin to absorb calcium and phosphate ions from the surrounding fluid, they grow spontaneously and they lead to the formation of chemical bonding between the bone and the implant. Titanium seems to have the same property, as hydroxyapatite layer may form on its surface in SBF environment. To the contrary, this is not the case for stainless steel and Cr- Co alloys (Kim et al., 1996).
In another trial (Kato et al., 2000), the bond between the bone and tantalum plates that had been thermally treated in alkaline environment was examined, in terms of mechanical and histological properties. The findings were compared to the findings of tantalum plates that had not undergone such treatment. In the first case, a bond between the plates and the bone could be detected within 16 weeks, whereas no such bond could be identified in the case of non-treated plates. Histologically, also, it could be seen that, again in the first case, there was direct contact between the plate and the bone. To the contrary, in the second case, fibrous tissue had developed in this interface.

The different reaction of the bone against pretreated porous tantalum, compared to non-pretreated, was explained later (Miyazaki et al., 2002), in terms of biochemistry. In this study, it was found that amorphous sodium tantalate forms on pretreated plates' surface and pretreatment through the already presented way is a prerequisite for this formation to happen. The formation of amorphous sodium tantalate rapidly leads to the formation of $\mathrm{Ta}-\mathrm{OH}$ groups on its surface, through the exchange of $\mathrm{Na}^{+}$ions to $\mathrm{H}_{3} \mathrm{O}^{+}$ions. It should be mentioned that the pretreatment of porous tantalum in a more alkaline environment $(5.0 \mathrm{M} \mathrm{NaOH})$ did not lead to hydroxyapatite formation on its surface, as the formation of Ta-OH groups could not be achieved, due to the high $\mathrm{Na}^{+}$concentrations that slowed down the already presented cation exchange (Miyazaki et al., 2000). The presence of Ta-OH groups is of great importance, as they interconnect to $\mathrm{Ca}^{++}$ions and subsequently, form a type of calcium tantalate, which reacts to phosphate anions that are present at the SBF environment. Finally, larger amounts of calcium and phosphate ions are absorbed, leading to hydroxyapatite formation (Miyazaki et al., 2002). Hydroxyapatite cores form at first, leading to the accumulation of calcium and phosphate and ending to the formation of a hydroxyapatite layer. This has also been shown in earlier studies (Ohtsuki et al., 1992). Apart from $\mathrm{Ta}-\mathrm{OH}$ groups, it is known that $\mathrm{Si}-\mathrm{OH}$ and $\mathrm{Ti}-\mathrm{OH}$ groups also induce the formation of hydroxyapatite, through their transformation to calcium silicate and calcium titanate respectively (Takadama et al., 2000; Nishiquchi et al., 2001). It has been noticed in the past that these groups are negatively charged in SBF environment (Parks, 1965; Li et al., 1994; Kosmulski, 1997). It is probably the negative charge that leads to the selective calcium absorption and subsequently, to hydroxyapatite formation. 
But apart from porous tantalum implants that have been treated in alkaline environment in order to induce hydroxyapatite formation on their surface after their implantation, it is equally interesting to assess the osseointegration of porous tantalum implants that are already covered by hydroxyapatite. In such a trial (Barrere et al., 2003a), porous tantalum cylinders where used. On their surface, Bone-like Carbonated Apatite (BCA) was formed, after being treated in a SBF environment. The thickness of BCA was $30 \mu \mathrm{m}$. These cylinders were then implanted in sheep's femoral bones and were removed after 6,12 or 24 weeks. In all cases, the BCA covered implants outperformed the noncovered ones in terms of bone integration. The bone showed an accelerated biologic response to the BCA covered implants, underlining the biologically active and osteoconductive nature of BCA coating. This study concluded that BCA covered porous tantalum implants lead to a faster and superior development of bone tissue when compared to non-covered ones. This results to a more stable fixation of the implant to the bone, something favorable in the case of implants that undergo weight-bearing.

A more recent study (Barrere et al., 2003b), again from the same center, examined the use of Octa-Calcium Phosphate (OCP) as a coating of porous tantalum implants. The findings of this study showed that OCP has a greater potential in terms of bone integration in comparison to BCA. Apart from that, the most impressive finding was that OCP might possess osteoinductive properties when covering porous surfaces, as it was found that it induced bone formation even when the implants were placed intramuscularly. This fact could be explained if considering OCP's rough surface, its slower resorption rate compared to BCA and probably, the presence of Bone Morphogenetic Proteins (BMPs) inside calcium phosphate crystals (Yuan, 2001; Ripamonti, 1996; Yuan et al., 1998). Further amelioration of calcium phosphate coatings may lead to an even better microscopic structure and an even slower resorption rate, allowing a more successful in vivo osseointegration of porous tantalum implants.

\subsection{Bone Tissue Formation}

As it has been found in the past (Bobyn et al., 1980), concerning bone formation on Cr-Co alloy implants and its mechanical properties, the ideal pore diameter is $50-400$ $\mu \mathrm{m}$. When tested, these implants had a maximum strength of $17 \mathrm{MPa}, 8$ weeks after their implantation. The same investigator, in following studies, assessed the bone formation inside structures of porous tantalum. Porous tantalum cylinders (Bobyn et al., 1999a) and acetabular implants (Bobyn et al., 1999b) were used. The findings were highly supportive for the use of porous tantalum.

In the case of the first study, porous tantalum cylinders with a size of $5 \times 10 \mathrm{~mm}$ were used. They were implanted to dog bones, crossing both bone cortices. Implants porosity was $75-80 \%$ and pore diameter was 430 and $650 \mu \mathrm{m}$. Cylinders with $430 \mu \mathrm{m}$ pore diameter were assessed at week 4, 16 and 52 postoperatively, whereas cylinders with $650 \mu \mathrm{m}$ were examined at week 2, 3, 4, 16 and 52. The samples were histologically examined. Also, mechanical testing was performed at week 4 and 16 in the case of implants with $430 \mu \mathrm{m}$ pore diameter. Bone tissue formation inside the implants is shown at Table 1. At week 4, bone tissue occupied $52.9 \%$ of pore volume of the implants with $650 \mu \mathrm{m}$ pore diameter. The same value for implants with $430 \mu \mathrm{m}$ pore diameter was $41.5 \%$. The difference between the two groups was considered as statistically significant. This difference remained statistically significant at week 16 , as the values were 69.2 and $63.1 \%$ respectively. To the contrary, at week 52, the difference was again statistically significant, but this time in favor of the group of the implants with a smaller pore size, as the values were $79.7 \%$ for this group and $70.6 \%$ for the other one. However, the practical and clinical significance of these differences is doubtful. The extensive bone formation in both groups is the most important finding and this should be kept in mind. So, if we consider the superior mechanical properties of porous tantalum with a smaller pore diameter, than this form of porous tantalum is, probably, more appropriate to use in the construction of implants.

The pattern of bone tissue formation inside the pores of porous tantalum was clarified through examination of specimens under electron microscopy. At week 2, bone was formed mainly at the drilling site and intramedullary. Although bone ingrowth was limited, there was bone formation close to porous tantalum trabeculae. At week 3, bone ingrowth could be detected, whereas, at week 4, bone tissue formation throughout the whole implant was a common finding. Finally, at week 16 and week 52, bone ingrowth was really dense and that was also the point for the bone-implant interface. Another interesting finding was the formation of Haversian systems and the activation of the procedure of bone remodeling inside the pores. 
Christos G. Paganias et al. / American Medical Journal 5 (2): 63-72, 2014

Table 1. Bone ingrowth in porous tantalum cylinders in relation to the time of examination and the value of pore diameter (Bobyn et al., 1999a, modified)

\begin{tabular}{|c|c|c|c|c|}
\hline \multirow[b]{2}{*}{ (week) } & \multicolumn{2}{|l|}{ Bone ingrowth } & \multicolumn{2}{|c|}{ Confidence interval } \\
\hline & $\begin{array}{l}\text { Time } \\
\text { diameter }\end{array}$ & $\begin{array}{l}430 \mu \mathrm{m} \text { pore } \\
\text { diameter }\end{array}$ & $\begin{array}{l}650 \mu \mathrm{m} \text { pore } \\
\text { (CI) } 95 \%\end{array}$ & $P$ value \\
\hline 2 & - & $13.3(\mathrm{n}=24)(95 \%$ CI $10.8-15.8)$ & - & - \\
\hline 3 & - & $23.0(\mathrm{n}=24)(95 \%$ CI $20.0-26.0)$ & - & - \\
\hline 4 & $41.5(\mathrm{n}=12)(95 \%$ CI $37.3-45.8)$ & $52.9(\mathrm{n}=23)(95 \%$ CI $50.4-55.4)$ & $6.9-15.9$ & 0.00003 \\
\hline 16 & $63.1(\mathrm{n}=18)(95 \%$ CI $58.2-68.0)$ & $69.2(\mathrm{n}=24)(95 \%$ CI $67.0-71.5)$ & $1.5-10.8$ & 0.01 \\
\hline 52 & $79.7(\mathrm{n}=24)(95 \%$ CI $76.9 \omega \zeta 82.5)$ & $70.6(\mathrm{n}=23)(95 \%$ CI $68.3 \omega \zeta 73.0)$ & $-12.9 \omega \zeta-5.4$ & 0.000008 \\
\hline
\end{tabular}

Mechanical testing revealed that, at week 4, the shear strength of the implant was, at least, $18.5 \mathrm{MPa}$. This is considered as a high value, compared to the shear strength obtained after 4 weeks of implantation for other porous materials. For example, at week 4, the shear strength of Cr-Co alloy was 9.3 MPa in a study under the same investigator (Bobyn et al., 1980), whereas in other studies it varies from 1.2-13.1MPa (Camron et al., 1976). Porous tantalum superior results could better be explained if we consider its higher porosity. Porous tantalum has a porosity of $75-80 \%$, whereas fiber mesh coating has a porosity of $40-50 \%$ and porous beads coating just $30-35 \%$. This means that there is more space inside the implant for bone tissue to form, leading to more favorable mechanical properties of the implant in terms of strength and of the time needed to achieve this strength after the implantation. The fast bone ingrowth seems to have major clinical importance, as it accelerates the procedure of implant's stabilization, thus allowing earlier full weight-bearing after surgery with the use of porous tantalum implants.

The same investigator studied, also, the patterns of bone ingrowth inside implants that underwent loading (Bobyn et al., 1999b). He studied a canine model, where he implanted acetabular monoblock prosthesis and observed them for 6 months. The bone-implant interface underwent radiological, histological and electron microscopy examination. The implant's fixation was found to be stable in all cases. In all cases bone ingrowth was observed. Bone ingrowth varied from 0.2-2 $\mathrm{mm}$ which is the maximum of space left for bone ingrowth, as the rest of the implant is occupied by polyethylene. These results are comparable to the ones of studies examining titanium fiber mesh (Pidhorz et al., 1993). Bone ingrowth was more intense and deeper at the periphery of the implant, probably because of the elliptical shape of the implant and the load concentration at the area. In average, $16.8 \%$ of the pores were occupied by bone tissue. The same value for the periphery of the implant was $25.1 \%$. These findings are comparable to those of older studies (Jasty et al., 1993), which studied the bone penetration inside prosthesis with a titanium fiber mesh coating or with a Cr-Co alloy porous beads coating. The values for these structures were 21.5 and $13.4 \%$ respectively. Apart from that, porous tantalum value is very close to cancellous bone density, which is $17.7 \%$. So the combination of porous tantalum high porosity with the good bone ingrowth that it shows is probably the reason for its superior mechanical strength.

Another serious finding was the filling of the gaps that were initially observed in some cases- as a result of inadequate reaming- between the implant and the acetabulum. The gaps were filled by new bone formation. This has been observed after the implantation of monoblock acetabular prosthesis in humans too (Macheras et al., 2006). It has been also observed in some cases of prosthesis with a titanium fiber mesh coating (Jasty et al., 1993), but to a lesser extend.

Potentially clinically significant was also the observation that fibrous tissue had formed inside the pores in areas that were not occupied by bone. This tissue might serve as a mechanical barrier that blocks debris from moving to the bone- implant interface.

What needs to be further clarified is the factor that plays the key role and leads to this extensive bone ingrowth inside porous tantalum implants. In vitro and in vivo studies have shown that bone formation is enhanced at the presence of rough, sandblasting- type, surfaces (Brunette, 1988; Goldberg et al., 1995; Hacking et al., 1999; Kieswetter et al., 1996a). As it is known, porous tantalum surface presents such a pattern and leads to extensive bone formation. So, probably, the role of its rough surface should be considered too.

\subsection{Osteoblast Differentiation and Functionality}

What has been already presented is the bone ingrowth inside porous tantalum. This is a common finding among many studies. What has not been studied that much and is of great interest, is the presence of osteoblasts inside the pores and furthermore, their potential for differentiation and their functionality.

When the first such study was conducted (Findlay et al., 2004), the surface of solid tantalum was compared to a 
surface of tantalum disposed through the CVD method- the method used in the fabrication of porous tantalum. These surfaces were also compared to the surfaces of the most common orthopedic metals and to the surface of Tissue Culture Plastic (TCP). This study concluded that tantalum is a suitable substrate for the proliferation and differentiation of osteoblasts. This fact is supported by the findings concerning the primary adhesion of osteoblasts to tantalum and their morphology after their adhesion. These findings do not differ from those of TCP, which is a benchmark in in vivo osteoblast study. Apart from that, the proliferation rate of the cells on tantalum surface was comparable to those observed for the other substrates. Expression of genes related to osteoblast function was also at the same levels for all substrates. What was studied is the expression of transcription factor CBFA1, of the extracellular proteins COL- 1 and OCN, of the cytokines IL-11, TNF- $\alpha$ and RANKL and of OPG. No statistically significant difference was found at the expression of these genes among the different substrates. The mineralization rate was found to be faster in the cases of solid and CVD tantalum, but the difference was not considered as statistically significant.

What is different in this study, compared to previous studies, is that the surface morphology of the substrates did not affect the function of osteoblasts. This could be partly expected, as, with the exception of CVD tantalum, all the surfaces were relatively smooth. As it has been already noticed, previous in vivo studies (Kieswetter et al., 1996b; Bigerelle et al., 2002; Keller, 1998; Schwartz et al., 2001) have shown that osteoblasts get more activated when they grow on rough surfaces. Probably, this could be explained if we keep in mind the borderline roughness of CVD tantalum surface, which means that it might not affect the proliferation and functionality of osteoblasts.

It should be mentioned, though, that this study examined the proliferation and functionality of osteoblasts on surfaces. In a following study (Welldon et al., 2008), the same investigators examined the same parameters for the osteoblasts, but, this time, in three dimensions. Concerning porous tantalum, the presence of osteoblasts could be detected everywhere inside the pores, independently of the depth of the area examined. At day 3, already, the cells seemed to adhere to trabeculae surface, after a few mitosis rounds. The mitotic process continued and reached a peek by day 14 and day 21 .

What was further examined is the degree of osteoblasts' differentiation. The expression of STRO-1 and alkaline phosphatise was measured, given that the expression of STRO-1 gets lower as the procedure of osteoblasts maturation progresses, whereas the expression of alkaline phoshatase gets higher (Gronthos et al., 1999).
It was concluded that the differentiation of osteoblasts inside the pores of porous tantalum took place faster, in comparison to other substrates and this could be seen even by day 14. This means that, probably, the presence of porous tantalum promotes osteoblasts differentiation.

One more finding enhancing the idea that the differentiation of osteoblasts is accelerated when they grow on a porous tantalum surface, was the level of expression of genes associated to the function of osteoblasts. At day 14, collagen type 1 (COL-1) and Bone Sialoprotein (BSP-1) gene expression was lower for osteoblasts cultivated on porous tantalum, compared to other substrates. The reduced expression of both genes reflects the reduction occurring during the procedure of osteoblasts differentiation (Stein and Lian, 1996), first for COL-1 and then for BSP-1, ending to the loss of expression of both of them by the time of maturation of osteoblasts (Aubin et al., 1995).

Finally, it should be mentioned that, at the same study (Welldon et al., 2008), the extension of in vitro mineralization of the substrates was examined. This was done at week 6 and the findings supported the idea that porous tantalum is, at least, the same effective as Tissue Culture Plastic (TCP) in inducing mineralization. What could not be clarified is the underlying reason that led to these results, as they could be the consequence of the fast proliferation of osteoblasts, or of their good functionality.

These findings are in accordance to the findings of a newer study (Justesen et al., 2009). In this study the morphology of osteoblasts was examined after being cultivated on different tantalum surfaces, with different topography. It was found that, the deeper the cells of the substrate surface were, the more elongated osteoblasts got. In previous studies (Schwartz et al., 2001; Dalby et al., 2003; Rice et al., 2003; Wojciak-Stothard et al., 1996) it was shown that the more elongated the shape of osteoblasts is getting, the more differentiated and more active these cells are. This means that, these substrates can serve as scaffolds, inducing bone formation inside their pores, no matter if they are implanted to the bone or not. Thus, they can be used not only as implants, but also as grafts.

\subsection{Differentiation and Functionality of Osteoblasts Derived from Elderly Women}

Orthopaedic implants are widely used in osteoporotic patients and also in difficult cases of patients with insufficient bone stock. Good results have been achieved by the use of porous tantalum implants not only in osteoporotic patients, but also in cases of patients with poor bone stock (Nelson et al., 2003), such as patients 
suffering from femoral head osteonecrosis (Shuler et al., 2007) and patients that have undergone radiotherapy (Rose et al., 2006). So, it is of great interest to compare osteoblasts taken from elderly patients with osteoblasts from younger patients in terms of proliferation and retention of their functionality inside porous tantalum.

For this reason, a study was undertaken (Sagomonyants et al., 2011). Osteoblasts originating from young women ( $<45$ years old) were compared to osteoblasts from elderly women ( $>60$ years old). As a substrate for cell culture, porous tantalum, titanium fibre mesh and Tissue Culture Plastic (TCP) were used. A first finding of this study was the fact that the adherence, the new bone matrix formation, the mineralization and the rate of proliferation observed for the cells derived from old women were lower compared to the same parameters of the cells of young patients. These findings are in accordance to previous ones, including the observation that there is over expression of genes related to cellular apoptosis in elderly patients (Zhou et al., 2008).

But, the most important finding of this study is the fact that porous tantalum induces new bone formation more extensively then titanium fiber mesh or tissue culture plastic, when talking about cultures of osteoblasts descending from elderly women. This could be mainly attributed to the fast cellular proliferation that takes place on the surface of porous tantalum. It is really interesting that osteoblast proliferation and new bone formation in cultures of cells derived from elderly women, when porous tantalum is present, is at least similar to that observed in cultures of cells from young women on the other substrates.

More specifically, the adherence of osteoblasts on porous tantalum was higher than that on the other substrates. This finding is in contrast to what has been already said (Welldon et al., 2008). However, the different age and sex group of patients that donated the cells and the different culture environment used could explain this difference. Porous tantalum better performance could be explained in terms of differences in the surface topography of the examined materials. Apart from this, older studies (Kieswetter et al., 1996a) have shown that osteoblast adherence depends not only on the microscopic texture of the surface of the material, but, also, on its physicochemical properties and on its surface energy. So, the rougher the surface is, the stronger the osteoblast adherence gets (Anselme et al., 2005; Lincks et al., 1998). This is, also, the fact for cellular proliferation. It seems that these processes are mediated by specific integrins (Gronowicz et al., 1996; Sinha et al., 1996) and it is hypothesized that porous tantalum microstructure induces osteoblast adherence through the production of these integrins. Even more, porous tantalum microstructure is of especially irregular pattern. Such surfaces present with a higher surface energy, something that makes them friendlier to new bone formation (Kieswetter et al., 1996b) and of course, the rougher the material is, the bigger its surface becomes, offering a wider area for the osteoblasts to adhere.

Apart from this, it is of great importance to mention the impact of porous tantalum on the proliferation rate of osteoblasts of both age groups (Fig. 1).

The proliferation rate that was observed on porous tantalum, for cells taken from young women, was 4 times higher compared to titanium fiber mesh and 12 times higher when compared to Tissue Culture Plastic (TCP). The findings for osteoblasts taken from old women were even more impressive, as the proliferation rate was 6 and 16 times higher respectively. These observations cannot be explained only in terms of increased adherence, as adherence is just $25-30 \%$ higher for porous tantalum compared to the other substrates. So it can be hypothesized that porous tantalum possesses inherent physicochemical properties that have an anabolic effect on osteoblast proliferation.

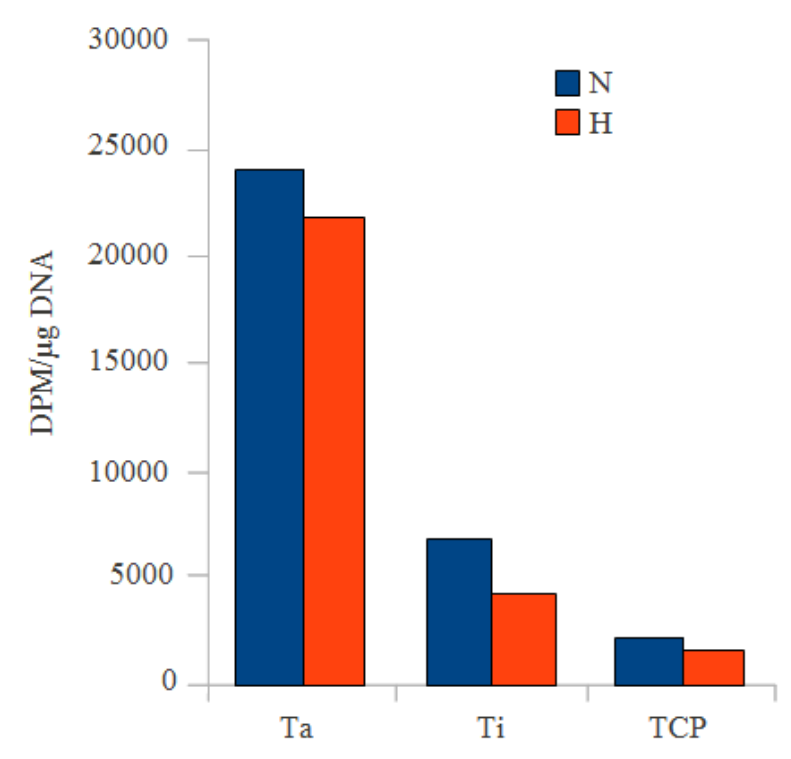

Fig. 1. Osteoblast proliferation rate in different materials cultures. (DPM: Disintegrations Per Minute). (Sagomonyants et al., 2011, modified) 
It is also interesting that porous tantalum had a different impact on the mineralization rate of bone matrix, depending on the age group examined. Whereas no significant difference was observed at week 3 between porous tantalum and titanium fiber mesh in the cultures of cells of young women, the difference was really significant among the cultures of osteoblasts of elderly patients, in favor of porous tantalum. This is an evidence for porous tantalum impact on cellular differentiation. Apart from this, one more fact that can serve as evidence is the expression of certain genes. At the same study (Sagomonyants et al., 2011), it was found that alkaline phosphatase and osteocalcin gene expression on porous tantalum was at the same levels for both age groups. This was not the case for titanium fiber mesh, as the expression of these genes was higher among the cultures of cells from young women. Given the fact that these proteins are synthesized during osteoblast differentiation, it can be judged that osteoblasts present with a similar differentiation potential, no matter the age group examined, when growing on porous tantalum, which is not true for other materials, such as titanium fiber mesh.

\section{CONCLUSION}

Even though these findings are really encouraging, there is still the question on the way porous tantalum induces osteoblast adherence, proliferation and mineralization in general and more importantly, in cases of elderly women. In previous studies, it has been shown that topographic features of titanium and subsequently, osteoblast biologic response to titanium can be seriously modified through small changes in titanium chemical composition (Anselme et al., 2005). On the other hand even though the findings of studies examining Tritanium (porous titanium with a porosity similar to that of porous tantalum) where encouraging, too, they were inferior to those observed with the use of porous tantalum (Frenkel et al., 2004; Ramappa et al., 2009). Consequently, it is speculated that both chemical and topographic porous tantalum properties contribute to the appearance of its special features. However, further research is required in order to shed light to the underlying mechanism that promotes this process, as porous tantalum is a really promising metal for the treatment of cases where bone defect is present.

\subsection{Author's Contributions}

All authors equally contributed in this work.

\subsection{Ethics}

This article is original and contains unpublished material. The corresponding author confirms that all of the other authors have read and approved the manuscript and no ethical issues involved.

\section{REFERENCES}

Anselme, K. and M. Bigerelle, 2005. Topography effects of pure titanium substrates on human osteoblast long-term adhesion. Acta Biomater., 1: 211-22. DOI: 10.1016/j.actbio.2004.11.009

Aubin, J.E., F. Liu, L. Malaval and A.K. Gupta, 1995. Osteoblast and chondroblast differentiation. Bone, 17: S77-S83. DOI: 10.1016/8756-3282(95)00183-E

Barrere, F., C.M. Van Der Valk, G. Meijer, R.A.J. Dalmeijer and K. De Groot et al., 2003. Osteointegration of biomimetic apatite coating applied onto dense and porous metal implants in femurs of goats. J. Biomed. Mater. Res. Part B: Applied Biomater., 67: 655-665.

DOI: $10.1002 / \mathrm{jbm} . b .10057$

Barrere, F., C.M. Van Der Valk, R.A.J. Dalmeijer, G. Meijer and C.A. Van Blitterswijk et al., 2003. Osteogenecity of octacalcium phosphate coatings applied on porous metal implants. J. Biomed. Mater. Res., 66A: 779-788. DOI: 10.1002/jbm.a.10454

Bigerelle, M., K. Anselme, B. Noël, I. Ruderman and P. Hardouin et al., 2002. Improvement in the morphology of Ti-based surfaces: A new process to increase in vitro human osteoblast response. Biomaterials, 23: 1563-1577.

DOI: 10.1016/S0142-9612(01)00271-X

Bobyn, J.D., G.J. Stackpool, S.A. Hacking, M. Tanzer and J.J. Krygier, 1999a. Characteristics of bone ingrowth and interface mechanics of a new porous tantalum biomaterial. J. Bone Joint Surg. Br., 81: 907-914.

Bobyn, J.D., K.K. Toh, S.A. Hacking, M. Tanzer and J.J. Krygier, 1999b. Tissue response to porous tantalum acetabular cups: A canine model. J. Arthroplasty, 14: 347-354. DOI: 10.1016/S0883-5403(99)90062-1

Bobyn, J.D., R.M. Pilliar, H.U. Cameron and G.C. Weatherly, 1980. The optimum pore size for the fixation of porous-surfaced metal implants by the ingrowth of bone. Clin Orthop., I50: 263-270.

Brunette, D.M., 1988. The effects of implant surface topography on the behavior of cells. Int. J. Oral. Maxillofac Implants, 3: 231-246. 
Camron, H.U., R.M. Pilliar and I. Macnab, 1976. The rate of bone ingrowth into porous metal. J. Biomed. Mater. Res., 10: 295-302.

DOI: $10.1002 / \mathrm{jbm} .820100210$

Dalby, M.J., M.O. Riehle, S.J. Yarwood, C.D. Wilkinson and A.S. Curtis, 2003. Nucleus alignment and cell signaling in fibroblasts: Response to a microgrooved topography. Exp. Cell Res., 284: 272-280. DOI: $10.1016 / \mathrm{S} 0014-4827(02) 00053-8$

Findlay, D.M., K. Welldon, G.J. Atkins, D.W. Howie and A.C. Zannettino et al., 2004. The proliferation and phenotypic expression of human osteoblasts on tantalum metal. Biomaterials, 25: 2215-2227.

DOI: 10.1016/j.biomaterials.2003.09.005

Frenkel, S.R., W.L. Jaffe, F. Dimaano, K. Iesaka and T. Hua et al., 2004. Bone response to a novel highly porous surface in a canine implantable chamber. J. Biomed. Mater. Res. B Appl. Biomater., 71: 387-391. DOI: 10.1002/jbm.b.30104

Geesink, R.G. and N.H. Hoefnagels, 1995. Six-year results of hydroxyapatite-coated total hip replacement. J. Bone Joint Surg. Br., 77: 534-547.

Goldberg, V.M., S. Stevenson, J. Feighan and D. Davy, 1995. Biology of Grit-Blasted Titanium Alloy Implants, Clin Orthop., 319: 122-129. PMID: 7554621

Gronowicz, G. and M. McCarthy, 1996. Response of human osteoblasts to implant materials: Integrinmediated adhesion. J. Orthop. Res., 14: 878-887. DOI: $10.1002 /$ jor.1100140606

Gronthos, S., A.C. Zannettino, S.E. Graves, S. Ohta, S.J. Hay et al., 1999. Differential cell surface expression of the STRO-1 and alkaline phosphatase antigens on discrete developmental stages in primary cultures of human bone cells. J. Bone. Miner Res., 14: 47-56. DOI: $10.1359 / j b m r .1999 .14 .1 .47$

Hacking, S.A., J.D. Bobyn, M. Tanzer and J.J. Krygier, 1999. The osseous response to corundum blasted implant surfaces in a canine hip model. Clin Orthop., 364: 240-253. PMID: 10416415

Jasty, M., C.R. Bragdon, T. Haire, R.D.M. Jr. and W.H. Harris, 1993. Comparison of bone ingrowth into cobalt chrome sphere and titanium fiber mesh porous coated cementless canine acetabular components. J. Biomed. Mater. Res., 27: 639-644. DOI: $10.1002 / \mathrm{jbm} .820270511$

Justesen, J., M. Lorentzen, L.K. Andersen, O. Hansen and J. Chevallier et al., 2009. Spatial and temporal changes in the morphology of preosteoblastic cells seeded on microstructured tantalum surfaces. J. Biomed. Mater. Res., 89: 885-894.

DOI: 10.1002/jbm.a.32032
Kato, H., T. Nakamura, S. Nishiguchi, Y. Matsusue and M. Kobayashi et al., 2000. Bonding of alkali- and heat-treated tantalum implants to bone. J. Biomed. Mater. Res., 53: 28-35. DOI: 10.1002/(SICI)10974636(2000)53:1<28::AID-JBM4>3.0.CO;2-F

Keller, J.C., 1998. Tissue compatibility to different surfaces of dental implants: In vitro studies. Implant Dent., 7: 331-337. PMID: 10196810

Kieswetter, K., Z. Schwartz, D.D. Dean and B.D. Boyan, 1996a. The role of implant surface characteristics in the healing of bone. Crit. Rev. Oral. Biol. Med., 7: 329-345. DOI: 10.1177/10454411960070040301

Kieswetter, K., Z. Schwartz, T.W. Hummert, D.L. Cochran and J. Simpson et al., 1996b. Surface roughness modulates the local production of growth factors and cytokines by osteoblast-like MG-63 cells. J. Biomed. Mater. Res., 32: 55-63.

DOI:

$10.1002 /($ SICI $) 1097$ 4636(199609)32:1<55::AID-JBM7>3.0.CO;2-O

Kim, H.M., F. Miyaji, T. Kokubo and T. Nakamura, 1996. Preparation of bioactive Ti and its alloys via simple chemical surface treatment. J. Biomed. Mater. Res., 32: 409-417.

DOI:

$10.1002 /($ SICI) 1097 4636(199611)32:3<409::AID-JBM14>3.0.CO;2-B

Kitsugi, T., T. Nakamura, M. Oka, Y. Senaha and T. Goto et al., 1996. Bone-bonding behavior of plasma-sprayed coatings of BioglassR, AW-glass ceramic and tricalcium phosphate on titanium alloy. J. Biomed. Mater. Res., 30: 261-269.

DOI:

$10.1002 /(\mathrm{SICI}) 1097$ 4636(199602)30:2<261::AID-JBM17>3.0.CO;2-P

Kokubo, T., 2004. Metallic materials stimulating bone formation. Med. J. Malaysia, 59: 91-2. PMID: 15468833

Kokubo, T., H. Kushitani, S. Sakka, T. Kitsugi and T. Yamamuro, 1990. Solutions able to reproduce in vivo surface-structure changes in bioactive glass-ceramic A-W3. J. Biomed. Mater. Res., 24: 721-734. DOI: $10.1002 / \mathrm{jbm} .820240607$

Kosmulski, M., 1997. Attempt to determine pristine points of zero charge of $\mathrm{Nb}_{2} \mathrm{O}_{5}, \mathrm{Ta}_{2} \mathrm{O}_{5}$ and $\mathrm{HfO}_{2}$. Langmuir, 13: 6315-6320. DOI: 10.1021/la970469g

Kotani, S., Y. Fujita, T. Kitsugi, T. Nakamura and T. Yamamuro et al., 1991. Bone bonding mechanism of $\beta$-tricalcium phosphate. J. Biomed. Mater. Res., 25: 1303-1315. DOI: 10.1002/jbm.820251010

Levine, B.R., S. Sporer, R.A. Poggie, C.J. Della Valle and J.J. Jacobs, 2006. Experimental and clinical performance of porous tantalum in orthopedic surgery. Biomaterials, 27: 4671-4681. DOI: 10.1016/j.biomaterials.2006.04.041 
Li, P., C. Ohtsuki, T. Kokubo, K. Nakanishi and N. Soga et al., 1994. The role of hydrated silica, titania and alumina in inducing apatite on implants. J. Biomed Mater Res., 28: 7-15. DOI: 10.1002/jbm.820280103

Lincks, J., B.D. Boyan, C.R. Blanchard, C.H. Lohmann and Y. Liu et al., 1998. Response of MG63 osteoblast-like cells to titanium and titanium alloy is dependent on surface roughness and composition. Biomaterials, 19: 2219-2232. DOI: 10.1016/S0142-9612(98)00144-6

Macheras, G.A., P.J. Papagelopoulos, K. Kateros, A.T. Kostakos and D. Baltas et al., 2006. Radiological evaluation of the metal-bone interface of a porous tantalum monoblock acetabular component. J. Bone. Joint Surg. Br., 88: 304-309.

DOI: 10.1302/0301-620X.88B3.16940

Miyazaki, T., H.M. Kim, F. Miyaji, T. Kokubo and H. Kato et al., 2000. Bioactive tantalum metal prepared by $\mathrm{NaOH}$ treatment. J. Biomed. Mater Res., 50: 35-42. DOI: $10.1002 /($ SICI) $1097-$ 4636(200004)50:1<35::AID-JBM6>3.0.CO;2-8

Miyazaki, T., H.M. Kim, T. Kokubo, C. Ohtsuki and H. Kato et al., 2002. Mechanism of bonelike apatite formation on bioactive tantalum metal in a simulated body fluid. Biomaterials, 23: 827-832. DOI: $10.1016 / \mathrm{S} 0142-9612(01) 00188-0$

Nelson, C.L., J.H. Lonner, A. Lahiji, J. Kim and P.A. Lotke, 2003. Use of a trabecular metal patella for marked patella bone loss during revision total knee arthroplasty. J. Arthroplasty, 18: 37-41. DOI: 10.1016/S0883-5403(03)00290-0

Nishiquchi, S., H. Kato, M. Neo, M. Oka and H.M. Kim et al., 2001. Alkali- and heat-treated porous titanium for orthopedic implants. J. Biomed. Mater Res., 54: 198-208. DOI: 10.1002/10974636(200102)54:2<198::AID-JBM6>3.0.CO;2-7

Ohtsuki, C., T. Kokubo and T. Yamamuro, 1992. Mechanism of apatite formation on $\mathrm{CaO}-\mathrm{SiO}_{2}-\mathrm{P}_{2} \mathrm{O}_{5}$ glasses in a simulated body fluid. J. Non-Cryst Solids, 143: 84-92.

DOI: $10.1016 / \mathrm{S} 0022-3093(05) 80556-3$

Parks, G.A., 1965. The isoelectric points of solid oxides, solid hydroxides and aqueous hydroxy complex systems. Chem. Rev., 65: 177-198.

DOI: $10.1021 / \mathrm{cr} 60234 \mathrm{a} 002$

Pidhorz, L.E., R.M. Urban, J.J. Jacobs, D.R. Sumner and J.O. Galante, 1993. A quantitative study of bone and soft tissues in cementless porous-coated acetabular components retrieved at autopsy. J. Arthroplasty, 8: 213-225. DOI: 10.1016/S0883-5403(09)80015-6
Ramappa, M.A., A. Bajwa, A. Kulkarni, I. McMurtry and A. Port, 2009. Early results of a new highly porous modular acetabular cup in revision arthroplasty. Hip. Int., 19: 239-244. PMID: 19876878

Rice, J.M., J.A. Hunt, J.A. Gallagher, P. Hanarp and D.S. Sutherland et al., 2003. Quantitative assessment of the response of primary derived human osteoblasts and macrophages to a range of nanotopography surfaces in a single culture model in vitro. Biomaterials, 24: 4799-4818. DOI: $10.1016 / \mathrm{S} 0142-9612(03) 00381-8$

Ripamonti, U., 1996. Osteoinduction in porous hydroxyapatite implanted in heterotopic sites of different animal models. Biomaterials, 17: 31-35. DOI: 10.1016/0142-9612(96)80752-6

Rose, P.S., M. Halasy, R.T. Trousdale, A.D. Hanssen and F.H. Sim et al., 2006. Preliminary results of tantalum acetabular components for THA after pelvic radiation. Clin. Orthop. Relat. Res., 453: 195-198. PMID: 17312592

Sagomonyants, K.B., M. Hakim-Zargar, A. Jhaveri, M.S. Aronow and G. Gronowicz, 2011. Porous tantalum stimulates the proliferation and osteogenesis of osteoblasts from elderly female patients. J. Orthop. Res., 29: 609-616. DOI: 10.1002/jor.21251

Schwartz, Z., C.H. Lohmann, M. Sisk, D.L. Cochran, V.L. Sylvia and J. Simpson et al., 2001. Local factor production by MG63 osteoblast-like cells in response to surface roughness and $1,25-(\mathrm{OH}) 2 \mathrm{D} 3$ is mediated via protein kinase $\mathrm{C}$ - and protein kinase Adependent pathways. Biomaterials, 22: 731-741. DOI: $10.1016 / S 0142-9612(00) 00241-6$

Shuler, M.S., M.D. Rooks and J.R. Roberson, 2007. Porous tantalum implant in early osteonecrosis of the hip: Preliminary report on operative, survival and outcomes results. J. Arthroplasty, 22: 26-31. DOI: $10.1016 /$ j.arth.2006.03.007

Sinha, R.K. and R.S. Tuan, 1996. Regulation of human osteoblast integrin expression by orthopedic implant materials. Bone, 18: 451-457. DOI: 10.1016/8756-3282(96)00044-0

Stein, G.S., J.B. Lian, J.L. Stein, A.J. Van Wijnen and M. Montecino, 1996. Transcriptional control of osteoblast growth and differentiation. Physiol. Rev., 76: 593-629.

Takadama, H., H.M. Kim, F. Miyaji, T. Kokubo and T. Nakamura, 2000. Mechanism of apatite formation induced by silanol groups. J. Ceram. Soc. Japan, 108: 118-121. DOI: 10.2109/jcersj.108.1254_118 
Welldon, K.J., G.J. Atkins, D.W. Howie and D.M. Findlay, 2008. Primary human osteoblasts grow into porous tantalum and maintain an osteoblastic phenotype. J. Biomed. Mater Res. A., 84: 691-701. DOI: $10.1002 / \mathrm{jbm} . \mathrm{a} .31336$

Wojciak-Stothard, B., A. Curtis, W. Monaghan, K. MacDonald and C. Wilkinson, 1996. Guidance and activation of murine macrophages by nanometric scale topography. Exp. Cell Res., 223: 426-435. DOI: $10.1006 /$ excr.1996.0098

Yuan, H., 2001. Osteoinduction of Calcium Phosphates. Leiden, ISBN-10: 909014417X
Yuan, H., P. Zou, Z. Yang, X. Zhang and J.D. De Bruijn et al., 1998. Bone morphogenetic protein and ceramic-induced osteogenesis. J. Mater Sci. Mater Med., 9: 717-721.

DOI: 10.1023/A:1008998817977

Zhou, S., J.S. Greenberger, M.W. Epperly, J.P. Goff, C. Adler et al., 2008. Age-related intrinsic changes in human bone-marrow-derived mesenchymal stem cells and their differentiation to osteoblasts. Aging Cell, 7: 335-343.

DOI: $10.1111 /$ j.1474-9726.2008.00377.x 